\title{
ADDRESS BY THE DIRECTOR GENERAL OF HIGHER EDUCATION MINISTRY OF EDUCATION AND CULTURE, REPUBLIC OF INDONESIA
}

Distinguished guests, and participants,

First of all I would like to second the Chairman of the Local Organizing Committee to welcome you in this very particular place here on the Island of Bali. Last night you already had the first experience of the richness of Balinese arts and dances, at the courtesy of our Governor of Bali. Of course you would want to know more about Bali and our cultural features of other regions as well. Our coat of arms mentions "Bhineka Tunggal Ika", meaning Unity in Diversity. This reflects the richness of our socio-economic and cultural heritage. With more than thirteen thousand islands that comprise the modern state of Indonesia, located at the crossroads of the Pacific Rim community, Indonesia indeed offers an endless variety of cultural life.

It is with great pleasure and anticipation that I am here to open the International Union Symposium No. 143 on Wolf-Rayet Stars and Interrelations with other Massive Stars in Galaxies. From what I have been informed, these types of stars are playing a very important role in modifying their galactic environments. In their short lifetime, of course on galactic time-scale, these stars have shown in their vigorous and efficient ways of revealing, that, at least according to some theories, nuclear reactions have actually taken place inside the stars. The products are brought out to the upper atmosphere, showing "bizarre" spectral features that attract the astronomers' interest. The relative youthness of the stars is important in many different fields of astronomy. They can, for example, be used to trace the spiral features of our Galaxy.

Indonesians live practically under the shadow of the center of the Milky Way, which we call Bimasakti, named after the ancient hero from the great Hindu epic Mahabharata. We are enchanted by the glamorous radiation from that part of the sky: the Milky Way band that stretches from South to North in our summer sky, which creates spectacular views since times immemorial. Many of us do not know what it is. It is gratifying, therefore, to learn that you in modern time, equipped with modern techniques and theoretical knowledge, are here to unravel one of the mysteries of the Bimasakti.

Here in Bali, where Hindu religion is predominant, life and death are viewed as an unending process. I wish all of you will be inspired by the wisdom of the Balinese, and will also find the studying of the life and death processes of stars in the galaxies a rewarding undertaking.

It is at this point that I would like to express our gratitudes, on behalf of the Government of Indonesia, that the International Astronomical Union has entrusted to one of our components of higher education to organize this important gathering. I hope that the Bali meeting will contribute substantially to the existing body of knowledge of astronomy. I would also like to express my sincerest thank to many organizations, here and abroad, that have made this meeting possible.

Finally, I wish you all a pleasant stay and fruitful meeting here on the Island of Paradise. Allow me to declare the beginning of the Symposium on Wolf-Rayet Stars and Interrelations with other Massive Stars in Galaxies.

Thank you.

Prof.Dr. Sukadji Ranuwihardjo

10

K. A. van der Hucht and B. Hidayat (eds.),

Wolf-Rayet Stars and Interrelations with Other Massive Stars in Galaxies, 10.

(c) 1991 IAU. Printed in the Netherlands. 